\title{
The power of cryo-electron tomography
}

\author{
Wolfgang Baumeister Max-Planck-Institute of Biochemistry
}

Traditionally, structural biologists have approached cellular complexity in a reductionist manner by characterizing isolated and purified molecular components. This 'divide and conquer' approach has been highly successful, as evidenced by the impressive number of entries in the PDB. In spite of this, awareness has grown in recent years that only rarely can a complex biological function be attributed to an individual macromolecule. Rather, most cellular functions arise from their acting in concert. Hence there is a need for methods developments enabling studies performed in situ, i.e. in unperturbed cellular environments. Sensu stricto the term 'structural biology in situ' should apply only to a scenario in which the cellular environment is preserved in its entirety. Electron cryotomography has unique potential to study the supramolecular architecture or 'molecular sociology' of cells. It combines the power of threedimensional imaging with the best structural preservation that is physically possible. Key methods developments, such as correlative LM/EM, Focussed Ion Beam Milling (cryo FIB) or phase plate imaging, will be discussed as well as applications highlighting the potential of this post-reductionist approach to structural biology. 